\title{
GROWTH RESULTS FOR A SUBCLASS OF BAZILEVIČ FUNCTIONS
}

\section{RABHA MD. EL-ASHWAH and D. K. THOMAS}

\author{
Department of Mathematics and Computer Science \\ University College of Swansea \\ Singleton Park \\ SWANSEA SA2 8PP \\ South Wales, U. K.
}

(Received March 29, 1985)

ABSTRACT. For $\alpha>0$, let $B(\alpha)$ be the class of regular normalized Bazilevic functions defined in the unit disc. Choosing the associated starlike function $g(z) \equiv z$ gives a proper subclass $B_{1}(\alpha)$ of $B(\alpha)$. For $B(\alpha)$, correct growth estimates in terms of the area function are unknown. Several results in this direction are given for $B_{1}\left(\frac{1}{2}\right)$.

KEY WORDS AND PHRASES. Bazilevic functions, subclasses of $s$, functions whose derivative has positive real part, close-to-convex functions, coefficient and length-area estimates.

1980 AMS SUBIECT CLASSIFICATION CODE. $30 C 45$.

1. INTRODUCTION.

Let $S$ be the class of regular, normalized, univalent functions with power series expansion

$$
f(z)=z+\sum_{n=2}^{\infty} a_{n} z^{n}
$$

for $z \in D$, where $D=\{z:|z|<1\}$.

Denote $R, S^{*}, K$ and $B(\alpha)$ the subclasses of $S$ which are functions whose derivative has positive real part [8], starlike with respect to the orgin [9 p.221], closeto-convex [6] and Bazilevic of type $\alpha$ [13] respectively. Following [13] we define $\mathrm{f} \in \mathrm{B}(\alpha), \quad \alpha>0$ to be the class of functions $\mathrm{f}$, regular and normalized in $\mathrm{D}$, such that, there exist $g \in S^{*}$ such that for $z \in D$,

$$
\operatorname{Re} \frac{z f^{\prime}(z)}{f(z)^{1-\alpha} g(z)^{\alpha}}>0 \text {. }
$$

Then if $g(z) \equiv f(z), \quad B(\alpha)=S^{*}$ and $B(1)=K$. Let $C(r)$ denote the closed curve which is the image of $D$ under the mapping $w=f(z), L(r)$ be the length of $C(r)$ and $A(r)$ the area enclosed by the curve $C(r)$. For $f \in S^{*}$, it was shown [7] that, with $z=r e^{i \theta}, 0<r<1$,

$$
L(r)=0(1)\left(M(r) \log \frac{1}{1-r}\right) \text { as } r \rightarrow 1 \text {, }
$$

where $M(r)=\max _{|z|=r}|f(z)|$, and Hayman [4] gave an example to show that this estimate is best possirle when $f$ is bounded. In [14] this result was extended to starlike func- 
tions with $A(r)<A$ constant. A modification of this nethod also shows that for $\mathrm{f} \in \mathrm{S}^{*}$,

$$
L(r)=O(1) \sqrt{ } A(r)\left(\log \frac{1}{1-r}\right) \text { as } \quad r \rightarrow 1 \text {. }
$$

Thomas [14] also showed that (1.3) holds for the class $K$ and for the class $B(\alpha)$, $0<\alpha \leq 1$ [13]. It is apparently an open question that (1.4) is valid for $f \epsilon K$ or $B(\alpha)$.

Pommerenke [11] showed that if $\mathrm{f} \in \mathrm{S}^{*}$, then for $\mathrm{n} \geq 2$

$$
\mathrm{n}\left|\mathrm{a}_{\mathrm{n}}\right| \leq \mathrm{C} \sqrt{\mathrm{A}}\left(\mathrm{l}-\frac{1}{\mathrm{n}}\right) \text {, }
$$

where $C$ is constant, and Noor [10] extended this to $B(\alpha)$ by showing that

$$
\mathrm{n}\left|\mathrm{a}_{\mathrm{n}}\right| \leq \mathrm{C} M\left(1-\frac{1}{\mathrm{n}}\right) \text {. }
$$

The question as to whether (1.5) is valid for $f \in K$ or $B(\alpha)$ is also apparently open.

In [12] the subclass $B_{1}(\alpha)$ of $B(\alpha)$ consisting of those functions in $B(\alpha)$ for which $g(z) \equiv z$ was considered and sharp estimates for the modules of the coefficients $a_{2}, a_{3}$, and $a_{4}$ were given. In [15] Thomas gave sharp estimates for the coefficients $a_{n}$ in (1.1) when $\alpha=1 / \mathrm{N}, \mathrm{N}$ a positive integer.

In this paper we shall be concerned with the class $B_{1}\left(\frac{1}{2}\right)$ and will use the method of Clunie and heogh [1] to establish (1.5) and hence (1.6) and the method of Thomas [14] to prove (1.4) and hence (1.3). The methods will in fact give results which are stronger for this subclass.

2. STATEMENTS OF MAIN RESULTS.

THEOREM 1. Let $\mathrm{f} \in \mathrm{B}_{1}\left(\frac{1}{2}\right)$ and be given by $(1.1)$. Then

$$
\begin{aligned}
& \text { (i) } n\left|a_{n}\right| \leq o(1)+0(1) \sqrt{ }\left(1-\frac{1}{n}\right), \text { as } n \rightarrow \infty \text {, } \\
& \text { (ii) } L(r)=0(1)\left\{\sqrt{ } A(r) \log \frac{1}{1-r}\right\} \quad \text { as } r \rightarrow 1 .
\end{aligned}
$$

We shall need the following:

LEMMA 1. Let $f \in B_{1}\left(\frac{1}{2}\right)$ and be given by (1.1). Define the function $F$ in $D$ by $F(z)^{2}=f\left(z^{2}\right)$. Then

PROOF. For $z=\rho \mathrm{e}^{\mathrm{i} \theta}, 0 \leq \mathrm{r}<1$,

$$
A(r, F) \leq \frac{1}{2(\pi-2)^{2}} A\left(r^{2}, f\right) .
$$

$$
\begin{aligned}
A(r, F) & =\int_{0}^{2 \pi} \int_{0}^{r}\left|F^{\prime}(z)\right|^{2} \rho \mathrm{d} \rho \mathrm{d} \theta \\
& =\int_{0}^{2 \pi} \int_{0}^{r}\left|\frac{\mathrm{zf}^{\prime}\left(\mathrm{z}^{2}\right)}{\mathrm{f}\left(\mathrm{z}^{2}\right)^{\frac{1}{2}}}\right|^{2} \rho \mathrm{d} \rho \mathrm{d} \theta .
\end{aligned}
$$

Now $\left|\frac{1}{f\left(z^{2}\right)}\right| \leq \frac{4}{(\pi-2)^{2}}[15]$ and so using (1.1) we have

$$
\begin{aligned}
A(r, F) & \leq \frac{4}{(\pi-2)^{2}} \int_{0}^{2 \pi} \int_{0}^{r}\left|z f^{\prime}\left(z^{2}\right)\right|^{2} \rho \operatorname{dod} \theta \\
& \left.=\frac{\pi}{2(\pi-2)^{2}} \sum_{n=1}^{\infty} n\left|a_{n}\right|^{2} r^{4 n}, \quad \text { (where }\left|a_{1}\right|=1\right) \\
& =\frac{1}{2(\pi-2)^{2}} A\left(r^{2}, f\right)
\end{aligned}
$$


LEMMA 2. For $\mathrm{f} \in \mathrm{S}$

$$
\begin{aligned}
& \text { (i) the map } r \rightarrow(1-r)^{6} A(r) /(1+r)^{2} r^{2} \text { is decreasing on the interval }(0,1) \text {, } \\
& \text { (ii) } A(\sqrt{ } r)<\frac{512}{r} A(r) \text { for } 0<r<1 .
\end{aligned}
$$

PROOF. Since

$$
A(r)=\int_{0}^{2 \pi} \int_{0}^{r}\left|f^{\prime}(z)\right|^{2} \rho d \rho d \theta
$$

we have

$$
\begin{aligned}
r A^{\prime}(r) & =\int_{0}^{2 \pi}\left|z f^{\prime}(z)\right|^{2} d \theta \\
\leq & 2 \int_{0}^{2 \pi} \int_{0}^{r}\left|f^{\prime}(z)\right|^{2}\left|\frac{z f^{\prime} '(z)}{f^{\prime}(z)}\right| \rho d \rho d \theta+2 \int_{0}^{2 \pi} f^{r}\left|f^{\prime}(z)\right|^{2} \rho d \rho d \rho .
\end{aligned}
$$

The classical distortion theorem for $f \in S[3, p .5]$ gives

$$
\begin{aligned}
r A^{\prime}(r) & \leq 4 r \frac{(r+2)}{1-r^{2}} \int_{0}^{2 \pi} \int_{0}^{r}\left|f^{\prime}(z)\right|^{2} \rho d \rho d \theta+2 \int_{0}^{2 \pi} \int_{0}^{r}\left|f^{\prime}(z)\right|^{2} \rho d \rho d \theta . \\
& =2 A(r)\left\{\frac{r^{2}+4 r+1}{1-r^{2}}\right\} .
\end{aligned}
$$

Thus

$$
\frac{d}{d r}(\log A(r)) \leq \frac{d}{d r}\left\{\left(\log \frac{r^{2}(1+r)^{2}}{(1-r)^{6}}\right)\right\}
$$

and part (i) of Lemma 2 is now obvious. Part (ii) follows immediately. PROOF OF THEOREM 1 .

(i) Since $f \in B_{1}\left(\frac{1}{2}\right)$, we can write from (1.2)

$$
z f^{\prime}\left(z^{2}\right)=f\left(z^{2}\right)^{\frac{1}{2}} h\left(z^{2}\right)
$$

where $\operatorname{Re} h(z)>0$, for $z \in D$.

Set

$$
h\left(z^{2}\right)=\frac{1+w\left(z^{2}\right)}{1-w\left(z^{2}\right)}
$$

where $w\left(z^{2}\right)$ is regular, $\left|w\left(z^{2}\right)\right|<1$ in $D, w(0)=0$ and $w(z)=\sum_{n=1}^{\infty} w_{n} z^{n}$. Then with $F(z)=f\left(z^{2}\right)^{\frac{1}{2}}$ and

$$
\begin{aligned}
& F(z)=z+\sum_{n=2}^{\infty} b 2 n-1^{2 n-1},(2.1) \text { gives } \\
& \left(z f^{\prime}\left(z^{2}\right)+F(z)\right) w\left(z^{2}\right)=z f^{\prime}\left(z^{2}\right)-F(z) .
\end{aligned}
$$

Thus

$$
\left\{2 z+\sum_{k=2}^{\infty}\left(k a_{k}+b_{2 k-1}\right) z^{2 k-1}\right\} w\left(z^{2}\right)=\sum_{k=2}^{\infty}\left(k a_{k}-b_{2 k-1}\right) z^{2 k-1} .
$$

Equating coefficients of $z^{2 n-1}$ in (2.2), we find that for $n \geq 2$

$$
n a_{n}-b_{2 n-1}=2 w_{n-1}+\left(2 a_{2}+b_{3}\right) w_{n-2}+\ldots+\left[(n-1) a_{n-1}+b_{2 n-3}\right] w_{1} .
$$

This means that the coefficient combination $n a_{n}-b_{2 n-1}$ on the left hand side of (2.2) depends only on the coefficient combinations $\left[2 a_{2}+b_{3}\right], \ldots,\left[(n-1) a_{n-1}+b_{2 n-3}\right]$ on the right-hand side. Hence, for $n \geq 2$ we can write

$$
\left\{2 z+{ }_{k=2}^{n-1}\left(k a_{k}+b_{2 k-1}\right) z^{2 k-1}\right\} w\left(z^{2}\right)=\sum_{k=2}^{n}\left(k a_{k}-b_{2 k-1}\right) z^{2 k-1}+\sum_{k=n+1}^{\infty} c_{k} z^{2 k-1}
$$


say. Squaring the moduli of both sides of (2.3) and integrating round $|z|=r$, we obtain, using the fact that $\left|w\left(z^{2}\right)\right|<1$ for $z \in D$,

$$
\begin{gathered}
\sum_{k=2}^{n}\left|k a_{k}-b_{2 k-1}\right|^{2} r^{4 k-2}+\sum_{k=n+1}^{\infty}\left|c_{k}\right|^{2} r^{4 k-2} \\
<4+\sum_{k=2}^{n-1}\left|k a_{k}+b_{2 k-1}\right|^{2}
\end{gathered}
$$

letting $r \rightarrow 1$, we have

$$
\sum_{k=2}^{n}\left|k a_{k}-b_{2 k-1}\right|^{2} \leq 4+\sum_{k=2}^{n-1}\left|k a_{k}+b_{2 k-1}\right|^{2} .
$$

Thus

$$
\left|n a_{n}-b_{2 n-1}\right|^{2} \leq 4+{ }_{k=2}^{n-1} k\left|a_{k}\right|\left|b_{2 k-1}\right|
$$

(where $\left|a_{1}\right|=\left|b_{1}\right|=1$ ).

Hence

$$
\begin{aligned}
\mid n a_{n} & -\left.b_{2 n-1}\right|^{2} \leq 4\left({ }_{k}^{n=1} k\left|a_{k}\right|^{2}\right)^{\frac{1}{2}}\left(\sum_{k=1}^{n=1} k\left|b_{2 k-1}\right|^{2}\right)^{\frac{1}{2}} \\
& \leq 4 r^{-4 n+1}\left({ }_{k=1}^{\infty} k\left|a_{k}\right|^{2} r^{4 k}\right)^{\frac{1}{2}}\left(\sum_{k=1}^{\infty}(2 k-1)\left|b_{2 k-1}\right|^{2} r^{4 k-2}\right)^{\frac{1}{2}}
\end{aligned}
$$

for $0<\mathrm{r}<1$, and so

$$
\left|n a_{n}-b_{2 n-1}\right|^{2} \leq 4 r^{-4 n+1} \sqrt{ }\left(\frac{A\left(r^{2}, f\right)}{\pi}\right) \quad \sqrt{ }\left(\frac{A(r, F)}{\pi}\right) .
$$

Lemma 1 now gives

$$
\left|n a_{n}-b_{2 n-1}\right|^{2} \leq \frac{4}{\sqrt{2 \pi(\pi-2)}} r^{-4 n+1} A\left(r^{2}, f\right) .
$$

and choosing $r^{2}=1-\frac{1}{n}$ we obtain

$$
\left|\mathrm{na}_{\mathrm{n}}-\mathrm{b}_{2 \mathrm{n}-1}\right|^{2} \leq \mathrm{CA}\left(1-\frac{1}{n}\right) \text {, where } \mathrm{C} \text { is constant. }
$$

Finally, it is easy to see that from the definition of $B_{1}\left(\frac{1}{2}\right), F \varepsilon R$ and so [8] for $\mathrm{n} \leq 2, \quad\left|\mathrm{~b}_{2 \mathrm{n}-1}\right| \leq \frac{2}{2 \mathrm{n}-1}$. Thus (2.5) gives

$$
n\left|a_{n}\right|=o(1)+0(1) V\left(A\left(1-\frac{1}{n}\right)\right) \text { as } n \rightarrow \infty \text {. }
$$

This proves part (i) of Theorem 1 .

(ii)

$$
\text { Since } \begin{aligned}
L(r) & =\int_{0}^{2 \pi}\left|z f^{\prime}(z)\right| d \theta \text {, and } F(z)^{2}=f\left(z^{2}\right),(2.1) \text { gives } \\
L & =\int_{0}^{2 \pi}\left|z^{2} f^{\prime}\left(z^{2}\right)\right| d \theta \leq r \int_{0}^{2 \pi}\left|F(z) h\left(z^{2}\right)\right| d \theta \\
& \leq \int_{0}^{2 \pi} f^{r}\left|F^{\prime}(z) h\left(z^{2}\right)\right| \operatorname{d\rho d} \theta+2 r \int_{0}^{2 \pi} \int_{0}^{r}\left|F(z) h^{\prime}\left(z^{2}\right)\right| \rho d \rho d \theta \quad\left(z=\rho e^{i \theta}\right) \\
& =I_{1}(r)+I_{2}(r) \text { say. }
\end{aligned}
$$

Again using (2.1) we have

$$
I_{1}(r)=r \int_{0}^{r} \int_{0}^{2 \pi}\left|h\left(z^{2}\right)\right|^{2} d \theta d \rho=2 \pi r \int_{0}^{r}\left(1+{ }_{n=1}^{\infty}\left|h_{n}\right|^{2} \rho^{4 n}\right) d \rho
$$

where $h(z)=1+\sum_{n=1}^{\infty} h_{n} z^{n}$ for $z \in D$, and since $\left|h_{n}\right| \leq 2$ for $n \geq 1[2 p \cdot 10]$, 
GROWTH RESULTS FOR A SUBCLASS OF BAZILEVIC $\stackrel{\vee}{C}$ FUNCTIONS

$$
\begin{aligned}
I_{1}(r) & \leq 2 \pi r \int_{0}^{r}\left(1+4 \sum_{n=1}^{\infty} \rho^{4 n}\right) d \rho \\
& =0(1) \log \left(\frac{1}{1-r}\right) \text { as } r \rightarrow 1 .
\end{aligned}
$$

Also

$$
\begin{aligned}
& I_{2}(r) \leq 2 r\left(\int_{0}^{r} f_{0}^{2 \pi}\left|F(z)^{2} h^{\prime}\left(z^{2}\right)\right| \rho d \theta d \rho\right)^{\frac{1}{2}}\left(\int_{0}^{r} \int_{0}^{2 \pi}\left|h^{\prime}\left(z^{2}\right)\right| \rho d \theta d \rho\right)^{\frac{1}{2}} \\
& =2 \mathrm{r}\left(\mathrm{J}_{1}(\mathrm{r})\right)^{\frac{1}{2}}\left(\mathrm{~J}_{2}(\mathrm{r})\right)^{\frac{1}{2}} \text { say. }
\end{aligned}
$$

Since $\operatorname{Re} h\left(z^{2}\right)>0$, for $z \in D$, we may write

$$
h\left(z^{2}\right)=\frac{1}{2 \pi} \int_{0}^{2 \pi} \frac{1+z^{2} e^{-i t}}{1-z^{2} e^{-i t}} d \mu(t),
$$

where $\mu(t)$ increases and $\frac{1}{2 \pi} \int_{0}^{2 \pi} d \mu(t)=1$. [ 5 p. 68] .

Therefore

$$
h^{\prime}\left(z^{2}\right)=\frac{1}{\pi} \int_{0}^{2 \pi} \frac{e^{-i t}}{\left(1-z^{2} e^{-i t}\right)^{2}} d \mu(t),
$$

and so

$$
\left|h^{\prime}\left(z^{2}\right)\right| \leq \frac{1}{\pi} \int_{0}^{2 \pi} \frac{\mathrm{d} \mu(t)}{\left|1-\mathrm{z}^{2} \mathrm{e}^{-i t}\right|^{2}}
$$

Thus

$$
\mathrm{J}_{1}(\mathrm{r}) \leq \frac{1}{\pi} \int_{0}^{\mathrm{r}} \int_{0}^{2 \pi} \int_{0}^{2 \pi}\left|\frac{\mathrm{F}(\mathrm{z})}{1-\mathrm{z}^{2} \mathrm{e}^{-i t}}\right|^{2} \rho \mathrm{d} \theta \mathrm{d} \mu(\mathrm{t}) \mathrm{d} \rho .
$$

Since $F$ is an odd function we may write

$$
\frac{F(z)}{1-z^{2} e^{-i t}}=\sum_{n=1}^{\infty} S_{2 n-1}(t) z^{2 n-1}
$$

and so

$$
\int_{0}^{2 \pi} \int_{0}^{2 \pi}\left|\frac{F(z)}{1-z^{2} e^{-i t}}\right|^{2} d \theta d \mu(t)=2 \pi \sum_{n=1}^{\infty} \rho^{4 n-2} \int_{0}^{2 \pi}\left|S_{2 n-1}(t)\right|^{2} d \mu(t) .
$$

We now show that for $n \geq 1$,

$$
\int_{0}^{2 \pi}\left|s_{2 n-1}(t)\right|^{2} d \mu(t) \leq 2 \pi \sum_{j=1}^{n} j\left|a_{j}\right|\left|b_{2 j-1}\right|,
$$

where $F(z)=\sum_{n=1}^{\infty} b_{2 n-1} z^{2 n-1}$ for $z \in E$.

From (2.7) we have

$$
\sum_{n=1}^{\infty} S_{2 n-1}(t) z^{2 n-1}=\left(\sum_{n=1}^{\infty} b_{2 n-1} z^{2 n-1}\right)\left(\sum_{n=0}^{\infty} e^{-i n t} z^{2 n}\right)
$$

and so for $n \geq 1$,

$$
S_{2 n-1}(t)=\sum_{k=1}^{\infty} b_{2 k-1} e^{-i(n-k) t} .
$$

Now (2.1) gives

$$
\left(\sum_{n=1}^{\infty} n a_{n} z^{2 n-1}\right)=\left(\sum_{n=1}^{\infty} b_{2 n-1} z^{2 n-1}\right)\left(\sum_{n=0}^{\infty} h_{n} z^{2 n}\right)
$$

(where $h_{0}=1$ ) and so for $n \geq 1$

$$
n a_{n}=\sum_{\nu=1}^{n} b_{2 v-1} h_{n-v} \text {. }
$$

It is easy to see from (2.6) that, for $k \geq 1$, 


$$
h_{k}=\frac{1}{\pi} \int_{0}^{2 \pi} e^{-k i t} d \mu(t) .
$$

and so

$$
n a_{n}=\frac{1}{\pi} \sum_{\nu=1}^{n} b_{2 \nu-1} \int_{0}^{2 \pi} e^{-i(n-\nu) t}
$$

Now

$$
\begin{aligned}
\left|s_{2 n-1}(t)\right|^{2} & =s_{2 n-1}(t) \overline{s_{2 n-1}(t)} \\
& =2 \operatorname{Re} \sum_{j=1}^{n} k_{k=1}^{j} b_{2 j-1} \bar{b}_{2 k-1} e^{-i(k-j) t}-\sum_{v=1}^{n}\left|b_{2 v-1}\right|^{2}
\end{aligned}
$$

where we have used (2.8). Therefore, using (2.9)

$$
\begin{aligned}
\int_{0}^{2 \pi}\left|S_{2 n-1}(t)\right|^{2} d \mu(t) & =2 \operatorname{Re} j_{j=1}^{n} \sum_{k=1}^{j} b_{2 j-1} \bar{b}_{2 k-1} \int_{0}^{2 \pi} e^{-i(k-j) t} d \mu(t)-2 \pi \sum_{\nu=1}^{n}\left|b_{2 v-1}\right|^{2} \\
& =2 \pi \operatorname{Re}_{j=1}^{n} b_{2 j-1} \overline{j a}_{j}-2 \pi \sum_{\nu=1}^{n}\left|b_{2 v-1}\right|^{2} \\
& \leq 2 \operatorname{Re} \sum_{j=1}^{n} j \bar{a}_{j} b_{2 j-1} \\
& \leq 2 \pi \sum_{j=1}^{n}\left|a_{j}\right|\left|b_{2 j-1}\right| .
\end{aligned}
$$

Thus

$$
\begin{aligned}
J_{1}(r) & \leq 4 \pi f_{n}^{r} \sum_{n=1}^{\infty} \rho^{4 n-1}\left(\sum_{j=1}^{n} j\left|a_{j}\right|\left|b_{2 j-1}\right|\right) d \rho \\
& \leq 4 \pi \int_{0}^{r} \sum_{n=1}^{\infty} \rho^{4 n-1}\left(\sum_{j=1}^{n} j\left|a_{j}\right|^{2}\right)^{\frac{1}{2}}\left(\sum_{j=1}^{n} j\left|b_{2 j-1}\right|^{2}\right)^{\frac{1}{2}} d \rho \\
& \leq 4 \pi \int_{0}^{r} \sum_{n=1}^{\infty} \rho^{n} \sum_{j=1}^{\infty} i\left|a_{j}\right|^{2} \rho^{2 . j} \frac{1}{2} \quad \sum_{j=1}^{\infty}(2 j-1)\left|b_{2 j-1}\right|^{2} \rho^{4 j-2} \frac{1}{2} d \rho \\
& \leq 4 \int_{0}^{r} \frac{A(\rho, f)^{\frac{1}{2}} A(\rho, F)^{\frac{1}{2}}}{1-\rho} d \rho \\
& \leq \frac{4}{(\pi-2) \sqrt{2}} \int_{0}^{r} \frac{A(\rho, f)}{1-\rho} d \rho \text {, by lemma } 1 .
\end{aligned}
$$

Since $A(\rho)$ is increasing on $(0,1)$

Now

$$
\begin{aligned}
J_{1}(r) & \leq \frac{4}{(\pi-2) \sqrt{2}} A(r, f) \int_{0}^{r} \frac{d \rho}{1-\rho} \\
& =\frac{4}{(\pi-2) \sqrt{2}} A(r, f) \log \left(\frac{1}{1-r}\right) .
\end{aligned}
$$

$$
J_{2}(r)=\int_{0}^{r} \int_{0}^{2 \pi}\left|h^{\prime}\left(z^{2}\right)\right| \rho d \rho d \theta \text {, }
$$

and since $\quad\left|h^{\prime}\left(z^{2}\right)\right| \leq \frac{\operatorname{Re} h\left(z^{2}\right)}{1-r^{4}}$ for $0 \leq r \leq 1$,

$$
\begin{aligned}
J_{2}(r) & \leq 2 \int_{0}^{r} \int_{0}^{2 \pi} \frac{\operatorname{Re} h\left(z^{2}\right)}{1-\rho^{4}} \rho d \theta d \rho \\
& \leq 4 \pi \int_{0}^{r} \frac{d \rho}{1-\rho^{4}}
\end{aligned}
$$

since $h$ is harmonic in $D$.

Thus

$$
J_{2}(r) \leq 4 \pi \log \frac{1}{1-r} .
$$


Combining the estimates for $J_{1}(r)$ and $J_{2}(r)$ shows that

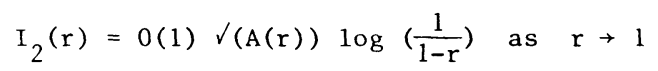

and the result is proved.

COROLLARY 1. Let $f \in B_{1}\left(\frac{1}{2}\right)$, then as $n \rightarrow \infty$,

(i) $n\left|a_{n}\right| \leq o(1)+O(1) P\left(1-\frac{1}{n}\right)^{\frac{1}{2}}$,

where for $0 \leq r<1, P(r)=\sum_{n=1}^{\infty}\left|a_{n}\right| r^{n}$

(ii) $n\left|a_{n}\right| \leq o(1)+0(1) A\left(1-\frac{1}{n}\right)^{\frac{1}{4}}(\log n)^{\frac{1}{4}}$,

(iii) $\quad \mathrm{L}(\mathrm{r})=0(1) \mathrm{A}(\mathrm{r})^{\frac{1}{4}}\left(\log \left(\frac{1}{1-\mathrm{r}}\right)\right)^{\frac{\mathrm{s}}{4}}$ as $\mathrm{r} \rightarrow 1$.

PROOF. From (2.4) and the fact that $\left|b_{2 k-1}\right| \leq \frac{2}{2 k-1}$ for $k \geq 1$, it follows that, for $0<r<1$,

$$
\begin{aligned}
\left|n a_{n}-b_{2 n-1}\right|^{2} & \leq 8 \underset{k=1}{n-1} \frac{k\left|a_{k}\right|}{2 k-1} \\
& \leq 4 r^{-n} P(r) .
\end{aligned}
$$

Choosing $r=1-\frac{1}{n}$ (i) follows.

(ii) follows since

$$
P(r) \leq\left(\sum_{n=1}^{\infty} n\left|a_{n}\right|^{2} r^{n}\right)^{\frac{1}{2}}\left(\sum_{n=1}^{\infty} \frac{r^{n}}{n}\right)^{\frac{1}{2}} .
$$

It follows trivially from(2.1) that

$$
L(r)=O(1) M(r)^{\frac{1}{2}} \log \frac{1}{1-r} \text { as } r \rightarrow 1
$$

and so (iii) follows at once on noting that

$$
M(r)^{2} \leq \frac{A(V r)}{\pi} \log \frac{1}{1-r}
$$

and on using lemma 2 .

REMARK. In view of Theorem 1 and Corollary 1 , it is possible that for $f \in B_{1}\left(\frac{1}{2}\right)$ the following conjectures are valid.

$$
\begin{aligned}
& \text { (i) } n^{2} a_{n}^{2}=0 \text { (1) } M\left(1-\frac{1}{n}\right) \quad \text { as } n \rightarrow \infty \text {, } \\
& \text { (ii) } \left.n^{4} a_{n}^{4}=0 \text { (1) A(1- } \frac{1}{n}\right) \quad \text { as } n \rightarrow \infty \text {, } \\
& \text { (iii) } \mathrm{L}(\mathrm{r})=0(\mathrm{l})(\mathrm{A}(\mathrm{r}))^{\frac{1}{4}} \log \left(\frac{1}{1-\mathrm{r}}\right) \text { as } \mathrm{r}+1 \text {. }
\end{aligned}
$$

We note that (ii) is stronger that (i) and that we have proved (ii) and (iii) in the case when $A(r)$ is finite.

The following extensions to Theorem 1 support the above conjectures.

3. INTEGRAL MEANS.

For $f$ regular in $D$, define for $\lambda$ real,

$$
I_{\lambda}(r, f)=\int_{0}^{2 \pi}\left|f\left(r e^{i \theta}\right)\right|^{\lambda} d \theta \text {. }
$$


THEOREM 2. For $\mathrm{f} \in \mathrm{B}_{\mathrm{l}}\left(\frac{1}{2}\right)$ and $\lambda>1$,

$$
I_{\lambda}\left(r^{2}, z f^{\prime}\right) \leq C(\lambda) \int_{0}^{r} \frac{M(\rho)^{\lambda / 2}}{(1-\rho)^{\lambda}} d \rho,
$$

where $C(\lambda)$ is a constant depending only on $\lambda$.

PROOF. (2.1) gives

$$
\begin{aligned}
I_{\lambda}\left(r^{2}, z f^{\prime}\right) & =\int_{0}^{2 \pi}\left|z^{2} f^{\prime}\left(z^{2}\right)\right|^{\lambda} d \theta \\
& \leq \lambda r \int_{0}^{r} \int_{0}^{2 \pi}\left|z f^{\prime}\left(z^{2}\right)\right|^{\lambda-1}\left|F^{\prime}(z) h\left(z^{2}\right)\right| d \theta d \rho \\
& +2 \lambda r \int_{0}^{r} \int_{0}^{2 \pi}\left|z f^{\prime}\left(z^{2}\right)\right|^{\lambda-1}\left|F(z) h^{\prime}\left(z^{2}\right)\right| \rho d \theta d \rho \\
& =J_{1}^{\prime}(r)+J_{2}^{\prime}(r) \text { say. }
\end{aligned}
$$

Now for $\lambda \geq 1$,

$$
J_{l}^{\prime}(r) \leq \lambda r \int_{0}^{r}\left(M\left(\rho^{2}, z f^{\prime}\right)\right)^{\lambda-1} \int_{0}^{2 \pi}\left|F^{\prime}(z) h\left(z^{2}\right)\right| d \theta \rho{ }^{1-\lambda} d \rho .
$$

From the proof of Theorem 1 (ii), we have with $z=\rho e^{i \theta}$,

$$
\int_{0}^{2 \pi}\left|F^{\prime}(z) h\left(z^{2}\right)\right| d \theta \leq 0(1) \frac{1}{1-\rho} \text { as } \rho \rightarrow 1 \text {. }
$$

Also (2.1) and the distortion theorem for functions of positive real part [4] gives

$$
M\left(\rho^{2}, z f^{\prime}\right) \leq \frac{2 \rho M(\rho, F)}{1-\rho} .
$$

Thus

$$
J_{1}^{\prime}(r) \leq C(\lambda) \int_{0}^{r} \frac{M(\rho, F)}{(1-\rho)^{-1}} d \rho
$$

and since $F(z)^{2}=f\left(z^{2}\right)$,

$$
J_{1}^{\prime}(r) \leq C(\lambda) \int_{0}^{r} \frac{M(\rho, f)^{\frac{\lambda-1}{2}}}{(1-\rho)^{\lambda}}
$$

Similarly, using the fact that $h$ is harmonic, we have

$$
\begin{aligned}
J_{2}^{\prime}(r) & \leq C(\lambda) \int_{0}^{r} \frac{M(\rho, F)^{\lambda}}{(1-\rho)^{\lambda}} \rho^{\lambda} d \rho \\
& \leq C(\lambda) \int_{0}^{r} \frac{M(\rho, f)^{\lambda / 2}}{(1-\rho) \lambda} d \rho
\end{aligned}
$$

Combining the results for $J_{1}^{\prime}(r)$ and $J_{2}^{\prime}(r)$ we obtain the result.

THEOREM 3. Let $\mathrm{f} \in \mathrm{B}_{1}\left(\frac{1}{2}\right)$, then for $0 \leq \mathrm{r} \leq 1$,

$$
I_{1}\left(r^{2}, f\right) \leq I_{1}\left(r^{2}, f_{0}\right)
$$

where

$$
f_{0}\left(z^{2}\right)=\left(\int_{0}^{z} \frac{1+t^{2}}{1-t^{2}} d t\right)^{2}
$$
PROOF. Since $f \in B_{1}\left(\frac{1}{2}\right)$, then $F \in R$.
Thus

$$
\begin{aligned}
\frac{1}{2 \pi} \int_{0}^{2 \pi}\left|f\left(z^{2}\right)\right| d \theta & =\frac{1}{2 \pi} \int_{0}^{2 \pi}|F(z)|^{2} d \theta \\
& =r^{2}+\sum_{n=2}^{\infty}\left|b_{2 n-1}\right|^{2} r^{4 n-2}
\end{aligned}
$$


and since for $n \geq 1,\left|b_{2 n-1}\right| \leq \frac{2}{2 n-1}$

$$
I_{1}\left(r^{2}, f\right) \leq r^{2}+4 \sum_{n=2}^{\infty} \frac{r^{4 n-2}}{(2 n-1)^{2}}=\frac{1}{2 \pi} \int_{0}^{2}\left|f_{0}\left(z^{2}\right)\right| d \theta
$$

and the theorem is proved.

\section{REFERENCES}

1. CLUNIE, J. and KEOGH, F. R., On starlike and schlicht functions, J. London Math. Soc. 35 (1960), 229-236.

2. GOODMAN, A. W., Univalent functions, Volume I 1983, Mariner Press, Florida.

3. HAYMAN, W. K., Multivalent functions, (Cambridge University Press 1958).

4. HAYMAN, W. K., On functions with positive real part, J. London Math. Soc. 36 (1961), 35-48.

5. HEINS, M., Selected topics in the classical theory of functions of a complex variable, (Holt, Reinhart, and Winston) (1962).

6. KAPLAN, W., Close-to-convex schlicht functions, Michigan Math. J. (1953)(52). 169185.

7. KEOGH, F. R., Some theorems on conformal mapping of bounded star-shaped domains. Proc. London Math. Soc. (3) 9 (1959).

8. MacGREGOR, T. H., Functions whose derivative has a positive real part, Trans. Amer. Math. Soc. 104 (1962), 532-537.

9. NEHARI, Z. Conformal Mapping, McGraw Hil1, 1952.

10. NOOR, I. K., Ph.D. thesis (1972), University College of Swansea, Wales.

11. POMERENKE, Ch., On Starlike and convex functions, J. London Nath. Soc. 37 (1962), 209-224.

12. SINGH, R., On Bazilevic functions, Proc. Amer. Math. Soc. 38, No. 2 (1973).

13. THOMAS, D. K., On Bazilevic functions, Trans. Amer. Math. Soc. 132, No. 2 (1968) 353-361.

14. THOMAS, D.K., On Starlike and close-to-convex univalent functions, J. London Math. Soc. 42(1967), 427-435.

15. THOMAS, D. K., On a subclass of Bazilevic functions. To appear. 


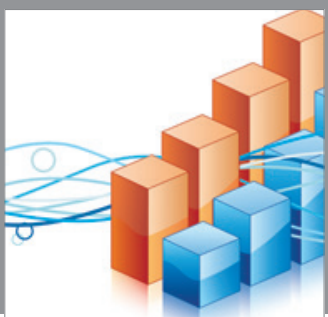

Advances in

Operations Research

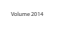

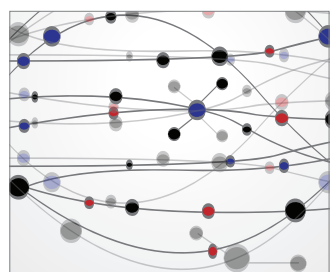

\section{The Scientific} World Journal
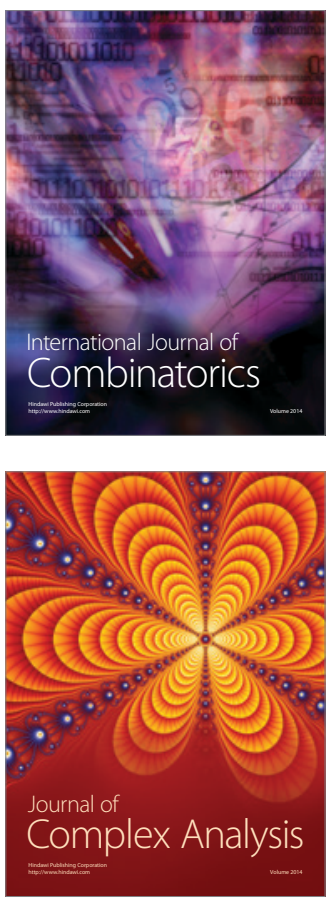

International Journal of

Mathematics and

Mathematical

Sciences
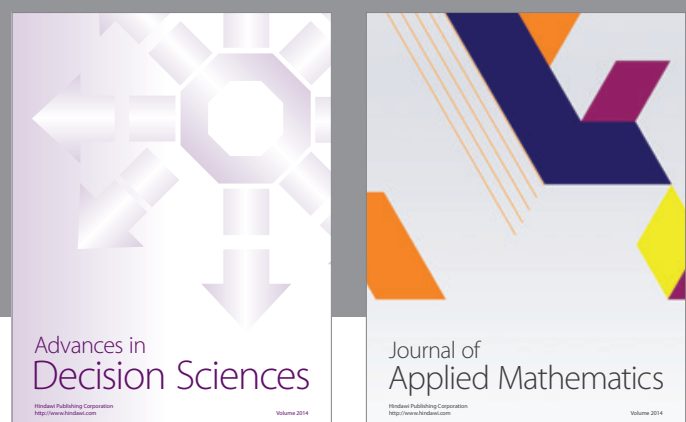

Journal of

Applied Mathematics
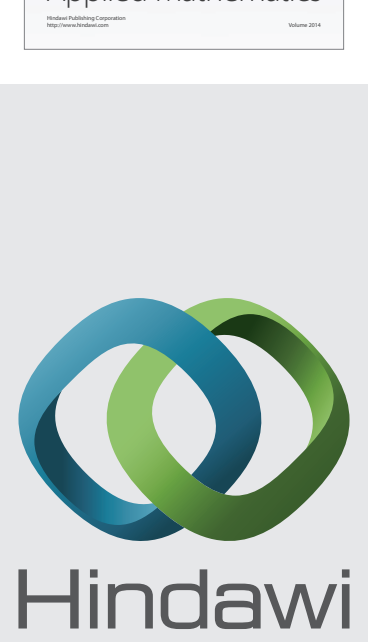

Submit your manuscripts at http://www.hindawi.com
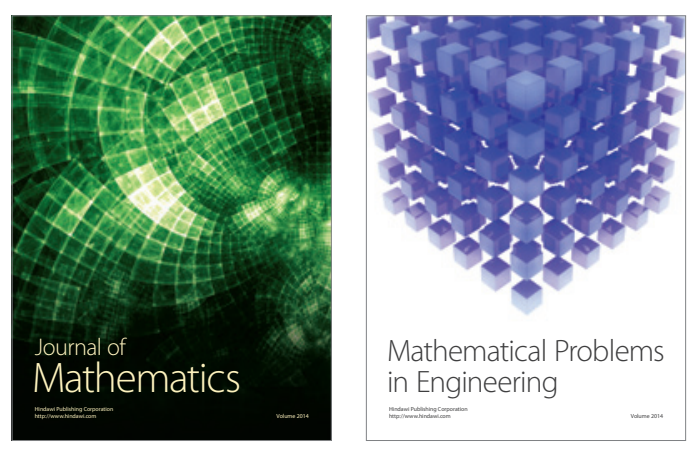

Mathematical Problems in Engineering
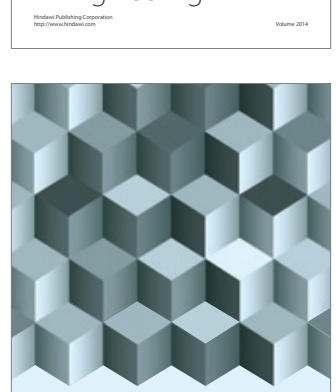

Journal of

Function Spaces
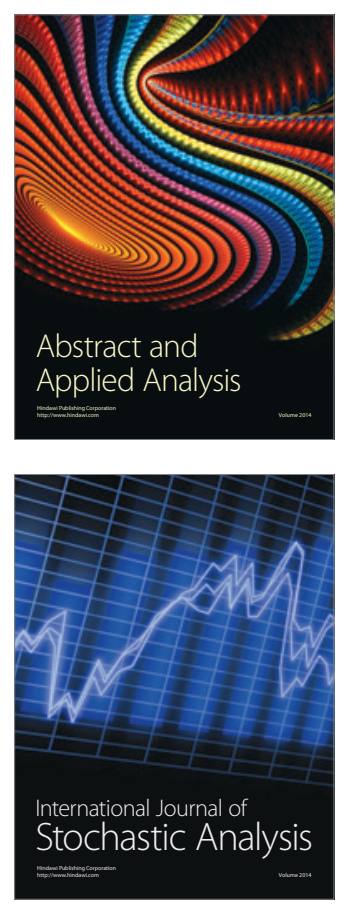

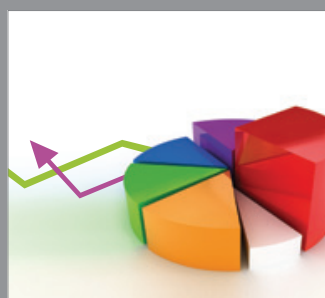

ournal of

Probability and Statistics

Promensencen
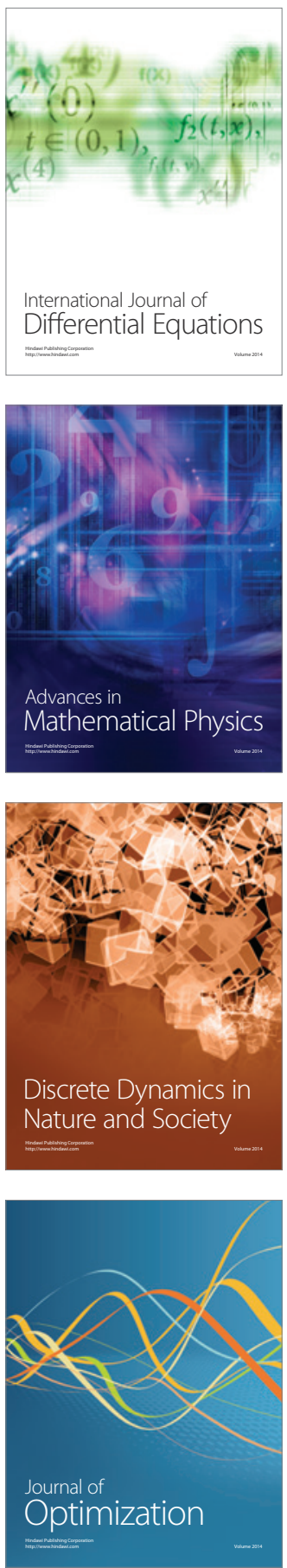